\title{
Coherent spectroscopic methods for monitoring pathogens, genetically modified products and nanostructured materials in colloidal solution
}

\author{
T. Moguilnaya ${ }^{1,2, *}$, Y. Suminov ${ }^{1}$, A. Botikov ${ }^{3}$, S. Ignatov $^{4}$, A. Kononenko ${ }^{5}$, and \\ A. Agibalov ${ }^{2}$ \\ ${ }^{1}$ JSC Giprogazoochistka, 105203 Moscow, Russia \\ ${ }^{2}$ JSC "SERVET-M", 115404 Moscow, Russia \\ ${ }^{3}$ Research Institute of Epidemiology and Microbiology, 123098 Moscow, Russia \\ ${ }^{4}$ State Research Center for Applied Microbiology and Biotechnology, 142279 Moscow, Russia \\ ${ }^{5}$ Veterinary Sanitation, Hygiene and Ecology Institute, 119991 Moscow Russia
}

\begin{abstract}
We developed the new automatic method that combines the method of forced luminescence and stimulated Brillouin scattering. This method is used for monitoring pathogens, genetically modified products and nanostructured materials in colloidal solution. We carried out the statistical spectral analysis of pathogens, genetically modified soy and nano-particles of silver in water from different regions in order to determine the statistical errors of the method. We studied spectral characteristics of these objects in water to perform the initial identification with $95 \%$ probability. These results were used for creation of the model of the device for monitor of pathogenic organisms and working model of the device to determine the genetically modified soy in meat.
\end{abstract}

We developed the coherent spectroscopic method for the monitoring of the pathogenic organisms directly in water pipe-line, genetically modified products and nanostructured materials in colloidal solution. The method is based on an analysis of spectral characteristics of stimulated radiation, passed through the solution.

We studied transmission IR spectra of a number of pathogens (salmonella, viruses of herpes genitaler, hepatitis A and C, grippe A and B ecd) and nanosilver solutions and luminescence of nanomarkers. In our experiments, the laser radiation passed through a quartz cell with water solutions of the pathogens, nanosilver or nanomarkers. Spectra were analyzed by the spectrum analyzer "Agilent" (USA) with spectral resolution $0.5 \mathrm{~nm}$. The solid DPSS lasers ("Laser-export Co. Ltd.", Russia) with wavelengths 1017, 810, 670, 532, $480 \mathrm{~nm}$ were used as the sources of exciting radiation. The input/output waveguides bring the exciting and passing radiations from laser to the cell and from cell to the analyzer, respectively.

We found that exciting radiations with wavelengths 1017 and $810 \mathrm{~nm}$ induce the stimulated Brillouin scattering in spectra of the water containing pathogen DNAs. We revealed that peak positions and widths of "fingerprints" for pathogens under study, and optical densities of these bands were proportional pathogen content, if their content was

\footnotetext{
*Corresponding author: mogilnay@mail.ru
} 
less $15 \%$. Thus, Stokes and anti-Stockes bands of the stimulated Brillouin scattering can be used to recognize the pathogens.

Nonlinear effects like SBS arising doe to the density of radiation in this solution [1]. The similar density of radiation is reached due to combination of the luminescence fields and laser radiation [2] giving the characteristic line. The modal frequency of this line depends on type DNA and accordingly from type of a pathogenic organism [3]. Similar lines are well interpreted by methods of the theory of recognition of images.

We have also established the new method for rapid analysis of genetically modified soy in meat products detected by a modified PCR fluorescent method. The transmission spectra of the pathogens, fluorescent markers, mix spectra (meat products, a soya) in a stationary solution and in the course of reaction have been investigated. It was found out that luminescence (around $1000-1300 \mathrm{~nm}$ ) was defined only PCR reaction, thus, parasitic radiation from spontaneous luminescence of albumin was minimum. Luminescence at 1500 $\mathrm{nm}$ is defined only for albumin of meat. It is shown that besides a signal of the fluorescent marker in the course of carrying out PCR of reaction, the own fluorescence of the soya exists at $1000 \mathrm{~nm}$. We have developed a working model of the device for the automated monitoring genetically modified sample soy. Based on these studies, we have developed the device for the automated control of pathogens in the water directly. We have also established the new method for rapid analysis of genetically modified products, representing a modified PCR fluorescent method.

The inventive methods can be recommended for DNA diagnosis, including field conditions, in medicine, veterinary sciences, in sanitary and epidemiological studies for detecting agents of dangerous infections, including potential bio-terrorist attacks.

It is of interest to develop this method to study changes in the composition under the influence of various factors. It was of interest to determine not only the content of the active cells, but also the content of destroyed cells. We are currently investigated spectral dynamics of invasive and inactivated pathogen. We measured the spectra of luminescence of live pathogens for several seconds, then the intensity of the luminescence decayed and we analyzed the peaks of the laser mode and the Stokes or anti-Stokes peaks. Stokes components were observed within $12-25$ min depending on the type of pathogen, then only the peak of the laser mode was observed. We did not detect luminescence inactivated pathogen. In case the dead pathogens, after a delay of $2-3 \mathrm{~min}$, the peak intensity of the Stokes component increased and the intensity of the laser mode decreased. We found that the intensity of the Stokes component for the dead bacterial cells was inactivated by several orders of magnitude to compare with alive bacterial cells toghether with reducing of the intensity of the laser mode.

We studied three pathogens - Staphylococcus aureus, Escherichia coli and coliphage MS-2. We found the Lamb dip in the peak corresponding to the Stokes and anti-Stokes component in all spectra of bacteriophages and did not reveal it in the virus spectrum.

We studied the influence of nanosilver on changes on the concentration of the live pathogens. After increase of the silver content, the peak was observed in the anti-Stokes field and the intensity decreased. We assume that this effect is associated with the death of pathogens. Standard biological method is qualitatively in agreement with our results.

We are currently investigated the spectral dynamics of particles of nanosilver depending on concentration. Spectral characteristics are changed at the nanoparticle concentration of $10 \mathrm{mg} / \mathrm{l}$. It is likely due to the aggregation of the particles.

\section{References}

1. T.Yu. Moguilnaya, E.A. Saguitova, A.G. Botikov, Proc. of SPIE 5621, 18 (2003)

2. T. Moguilnaya, P. Bobkov, V. Tomilin, J. Phys.: Conf. Ser. 414, 012023 (2013)

3. T. Moguilnaya et al., Pribory 1, 45 (2013) (in Russian)

4. T.Yu. Moguilnaya et al., J. Phys.: Conf. Ser. 594, 012026 (2015) 